\title{
Nurses' views on workplace wellbeing programmes
}

Nicola Wright, Melissa Zakarian, Holly Blake.

\begin{abstract}
Workplace stress is prevalent amongst nurses. Healthcare employers have implemented complementary and alternative therapies (CATs) for relaxation and stress management, within workplace wellbeing programmes for their employees. In-depth interviews were conducted with 12 registered nurses to explore the perceptions and experiences of nurses towards accessing CATs within and outside the workplace. Interviews were audio recorded and transcribed verbatim. Data were analysed using conventional, qualitative thematic techniques. Themes identified were "Perceptions of Complementary and Alternative Therapies for Stress Management" and "Engagement with Workplace Wellness Schemes". CATs have a role within workplace wellbeing programmes and nurses are not averse to accessing them, although there are barriers to access that need to be addressed.
\end{abstract}

Keywords: stress, nurses, complementary therapies, qualitative, workplace.

Key Phrases: (1) work-related stress is a challenge to public health; (2) amongst healthcare professions, nursing is amongst those with the highest levels of work related stress; (3) complementary and alternative therapies are a popular mechanism for stress relief; (4) nurses are not averse to using complementary and alternative therapies for workplace stress management. However, there are several logistical issues which impact on engagement with them.

\section{Introduction}

Work-related stress is a challenge to public health (Health and Safety Executive, 2014) and has a significant impact on organisations, since work environments shown to be stressful 
have higher levels of presenteeism and absenteeism (Wu et al., 2010) and a higher staff turnover rate (Begat et al., 2005). NHS Employers (2014) attributes 30\% of sickness in the UK National Health Service (NHS) to stress at an estimated cost of $£ 300-£ 400$ million per year.

Nursing has a high prevalence of work-related stress compared with other healthcare professions (Health and Safety Executive, 2014; Wu et al., 2010) and the reasons for this are multifaceted. Nursing demands a high level of skill, provision of 24-hour care and the input of what is referred to by Phillips (1996) as "emotional labour". Policy and organisational changes within the NHS influence priorities and mean that the nature of nursing work is in a constant state of flux (McVicar, 2003).

Given this context, healthcare organisations are increasingly implementing workplace wellness programmes to promote health and wellbeing amongst their employees (Blake et al, 2013; Lee et al, 2010; Lee and Blake, 2009). The organisational benefits of such interventions have been recognised (Lee et al, 2010; Blake and Lloyd, 2008), and evaluations indicate that employees are enthusiastic about workplace interventions for health, and perceive benefits from accessing them (Blake et al, 2015; Blake et al, 2014; Lee and Blake, 2009; Phillip and Thorne, 2008). Although diverse in nature, programmes often include exercise and fitness initiatives, dietary and healthy living advice and a range of complementary and alternative therapies (CATs) (Tiran and Chummun, 2004); CATs are the particular focus of this study. The National Centre of Complementary and Alternative Therapies (NCCAM) (2011) describe CATs as "a group of diverse medical and health care systems, practices and products that are not generally considered part of conventional medicine (NACCM, 2011 online). Such therapies may include massage, reflexology, reiki, acupuncture and herbal remedies. 
Despite high stress within nurses, workplace wellbeing initiatives are more commonly accessed by employees in office-based job roles than by front-line care staff, and evaluations of workplace programmes accessed by nurses are limited. Little is known about the perceptions of nurses towards the use of CATs for workplace wellbeing, although this knowledge is important for workplace health providers to better understand the needs of this employee group to inform the design of future services offered. Qualitative interviews with service users have shown that NHS employees (from diverse occupational groups) appreciate both the accessibility of complementary therapies offered through the workplace and the perceived benefits they bring to health and wellbeing (Meade et al, 2009). However, some nurses have expressed a lack of knowledge about CATs coupled with a degree of skepticism towards their use, which may influence their willingness to personally access them. Other factors may potentially hinder engagement, which are not well understood. The aim of this study was to explore nurses' perceptions and experiences of using CATs for workplace stress management.

\section{Methods}

An exploratory qualitative research design utilising in-depth, semi-structured interviews was conducted. Data collection took place between September 2011 and February 2012. All the interviews were conducted by the second author, lasted for between 20 and 60 minutes and were audio recorded. The data from the interviews were analysed using conventional qualitative thematic methods. These sought to identify themes both across and within individual accounts (Ritchie and Spencer, 1994).

Approval was obtained from the local NHS Research Ethics Committee (REF: 11/EM/0081) and research governance teams at the local hospital trust where the study took place. Verbal 
and written information was provided to all potential participants and informed written consent was obtained from those who agreed to be interviewed.

\section{Participants and Setting}

Participants were registered nurses recruited from the local Emergency Department and Burns High Dependency Unit of a single site of an acute hospital trust, using a combination of convenience and snowball sampling techniques. These settings were selected as it has been consistently evidenced that working in these clinical areas is stressful (Adriaenssens et al., 2015; Helps, 1997; Negble et al., 2014). Ward managers and research group leaders were asked to distribute a study information sheet to nurses within their teams. Those who wanted to participate returned an expression of interest to the researcher. A total of 12 registered nurses took part in the study. These included two prior users of the workplace therapies services who had accessed a Wellness Unit (SenzoriEgg) and massage therapies within the workplace setting, but infrequently accessed CATs outside of the workplace. The other 10 participants were non-users of the workplace therapies service, and eight of them had accessed a diverse range of CATs outside of the workplace setting, including massage therapies, acupuncture, aromatherapy, reflexology, and Reiki. Two participants had not previously accessed CATs. All of the participants that had accessed CATs, had accessed more than one type of CAT, whether inside or outside of the workplace. All participants were female and aged 24-53 years. They had worked at the hospital trust for between three and thirty-five years. Three of the nurses interviewed were Band Six deputy sisters and nine were Band Five registered nurses.

The NHS organisation where the study was undertaken has an established workplace wellness programme for employees, developed in 2005 and still active in 2016 at the time of publication. This offers a wide range of services to promote and support health and 
wellbeing, including staff gym, exercise classes and fitness challenges, staff physiotherapy service, and mental wellbeing (e.g. coping with stress and building resilience). Services have evolved over time and periodically change according to staff need but are described in more detail elsewhere (Blake et al, 2013) and in terms of CATs, have included a Wellness Unit (SenzoriEgg), massage, aromatherapy and reflexology. The range of workplace CATs available at the time of this study have been described in more detail elsewhere (Blake et al, 2013; Lee and Blake, 2009; Meade et al, 2009)

\section{Findings}

Analysis identified two key themes which were consistent both within and across the participant accounts. Identified themes and subthemes were agreed by a second person. Although participants were not given the opportunity to review their manuscripts, lay feedback and overal findings were circulated to all those registered with the service. The identified themes were: "perceptions of complementary therapies for stress management" and “engagement with workplace wellbeing initiatives". To preserve confidentiality and anonymity, participant numbers have been used for identification purposes.

\section{Theme 1: Perceptions of Complementary Therapies for Stress Management}

Before considering their involvement in workplace stress management initiatives, participants were asked to reflect on their perceptions and experiences of CATs more generally. All participants stated that they had engaged with them for perceived stress reduction with massage being the most popular form of therapy:

“...it just takes away the tension, really stops you feeling tense” (P004). 
Using CATs as a mechanism for managing stress was seen to be beneficial by the majority of participants, and several participants made references to the potential for CATs to reduce sickness absence in hospital employees when delivered in the workplace setting:

"I think it will reduce sickness, reduce people being irritable or upset on the wards" (P003).

However, some participants highlighted concerns about using CATs that appeared to be twofold; a lack of a robust evidence for the effectiveness of some therapies, coupled with a conflict between the underpinning philosophy of healthcare within the NHS (dominated by medicine) and the principles of some CAT approaches:

"It (CAT) has quite a stigma in this environment... it does, of being quite wishy washy, its about trying to correlate the two, medicine and complementary therapy" (P006).

For some, this conflict included a dissonance between their own cultural or spiritual beliefs and the philosophy perceived to underlie some CATs:

“I don't do yoga because it has a bit of a spiritual attachment to it...I'd rather not do it... because I don't want to compromise what I believe..." (P003).

In summary, the majority of participants had accessed CATs outside of the workplace, and perceived clear benefits of using CATs in the workplace to manage stress. However, the underpinning principles of some of the approaches was thought to conflict with both the dominant philosophy and evidence-based nature of healthcare provision and even personal belief systems. For some, this was identified as problematic for implementing CATs within the NHS workplace, and for their personal engagement with them.

Theme 2: Engagement with Workplace Wellbeing Initiatives 
Although all 12 participants were aware of workplace health initiatives within their local NHS organisation, only two had accessed them. One individual had accessed massages through the CATs service and the second had used the 'SenzOri Egg' (Lee and Blake, 2009). The latter is a self-contained wellness unit designed for taking 15 minute relaxation breaks. Both of the participants shared positive experiences of engaging in these activities:

"Massages are brilliant, I love it ... really helps" (P007).

“...on my night shifts sometimes on my break I would go and sit in the (SenzOri) egg, and it just kind of sits you back and the lights go dim and the door shuts around you, and it vibrates, its really cool and relaxing” (P010).

Those participants who had not used CATs in the workplace felt positively about their availability and recognised the potential benefits of CATs for stress reduction:

“...it makes sense to use them at work where we are most stressed” (P009).

"Going off to have a neck, back and shoulder massage would be lovely, definitely, it would refresh you, take your mind off work" (P011).

Nurses expressed that provision of CATs at work was a sign that the organisation valued its employees and that active steps were being taken to support their wellbeing:

"I think as a nurse you don't really expect to get anything, so the fact that you can have things like that subsidised, massages and things, is a really nice idea" (P009).

A number of logistical and practical barriers to access were raised, the most common being time, location and cost. Most notably, nurses perceived that they would not have the time to get away from the clinical setting in order to attend appointments for CATs: 
“My last ward, they didn't really have very good breaks, you didn't get the time...only had an hour and that wasn't really enough" (P006)

Although CATs services were designed to be accessible outside of shifts (e.g. on days off or at the end of the working day), nurses had little motivation to attend outside of their working hours:

“...it would have to probably be before or after a shift and then it would take some push to make me go, because at the end of the shift all you want is to go home" (P008).

Participants suggested that therapies could be delivered directly to them within their clinical setting to increase access and reduce time taken to engage in CATs:

"If they [the therapists] came on to the ward or something... and be there for us to just drop in when we have got a free minute.." (P009).

Cost of attendance was perceived to be prohibitive, although it was evident that participants were unaware that the cost of accessing workplace CATs was lower than average costs for therapies offered outside of the workplace:

“...they are quite expensive, they are about $£ 30$ - $\$ 40$ aren't they for half an hour... if I went for a massage every time I was stressed I think I'd be spending thousands" (P012).

However, nurses generally felt that the benefits of CATs would justify the cost:

"If I thought it would benefit me then I would pay it, definitely" (P013).

Issues were raised relating to the 'culture' of nursing as a profession, and for some, their nonengagement with workplace CATs was associated with logistical issues around attending and 
feeling unable to take a break at work. Many of the nurses felt that it would be perceived negatively if they took breaks at lunchtime, especially to attend a workplace health appointment: "staff didn't feel like they should go for breaks" (P006)

In summary, although participants were aware that CATs were offered within their workplace, knowledge about the provision was limited and very few of the nurses had personally accessed them, although their existence was enough to make nurses feel more valued by their employer. Nurses that had accessed the CATs perceived them to be highly beneficial. However, there were numerous barriers to access identified, including having the time to attend during the working day, location of services away from the clinical area, the misperception of high cost and a perception that their attendance for an appointment at the service would be perceived negatively by colleagues and superiors.

\section{Discussion}

In the UK, improving the health and wellbeing of National Health Service (NHS) employees remains high on public health agenda. A recent announcement by NHS England's Chief Executive Simon Stevens (NHS England, 2015) indicated a clear need to reduce stress in our public health workforce. The Emergency Department and Burns Units present particular challenges in this regard. The unique combination of acute (e.g. life or death decision making, violence and aggression) and chronic (e.g. high physical demands and time pressures) stressors with an unpredictability of working conditions can lead to nurses experiencing high levels of emotional distress and burnout (Adriaenssens et al., 2015). The consequences may impact on both the individual concerned, and the profession, since it has been identified that nurses experiencing these very intense reactions may change career, or use alcohol to cope with the stress (e.g. Duffy et al, 2015). Given the current shortage of nurses within the healthcare workforce (NHS Improvement, 2016) there is clearly a need to 
maintain a focus on supporting wellbeing and mangaging stress in this occupational group. In this study, we investigated nurse's views towards the use and implementation of CATs for managing stress at work and found their attitudes to be highly positive towards inclusion of such services within workplace wellness programmes, irrespective of whether they had accessed the services themselves.

At an organisational level, the provision of CATs within the workplace demonstrates that employers are concerned about staff welfare (Phillip and Thorne, 2008; Mackereth et al. 2005) and this resonates with the views expressed by our participating nurses. Indeed, prior evaluations have shown that staff opinions towards the NHS as an employer may be improved following implementation of workplace wellness programmes incorporating CATs (Blake et al., 2014).

Although participants identified the positive impact from treatments traditionally associated with CATs, it appeared that being able to access a quiet place if only for a few minutes was perceived to be very helpful in managing stress. This might simply be a quiet room in which nurses could take a break, or self-contained wellness units offering 'time-out' for nurses and potentially reducing perceived stress (Lee and Blake, 2009). Having a quiet place to go during breaks is not only perceived positively by individual nurses but may impact on patient care and outcomes. For example, Gardner et al. (2009) found that where 'quiet time' was implemented in clinical settings, patients and nurses felt more satisfied and relaxed with patients reporting improved sleep patterns.

Nurses from high-stress clinical environments, such as the Emergency Department and Burns Units, valued workplace-delivered CATs and perceived CATs to be beneficial to wellbeing, although some identified uncertainty and/or stigma associated with their use by healthcare 
professionals, which has been recognised previously (Adam, 2006; Furnham, 2001; Peters; 2000) and is often associated with a lack of robust evidence-base (Adam, 2006).

Where relevant, workplace health and wellbeing services need to increase awareness of the reduced cost of in-house CATs compared with external therapy services. Service providers should discuss alternative delivery times with nurses, and perhaps offer brief CATs that may be perceived to be more accessible. Nurses perceived a cultural barrier to taking work breaks and felt that accessing workplace wellness services within breaks would not be supported within their work environment. Managers and individual nurses need to be fully informed about the risks and costs associated with high stress within the nursing profession, and the potential benefits of workplace-delivered initiatives to prevent and manage stress. This may require cultural shift within hospital workplace environments to ensure that efforts to promote positive health and wellbeing for nurses is fully recognised by all, and where possible, break times for nurses are protected.

When considering the implications of the study it is important to do so within the context of its limitations. Data collection took place in 2011/2012 and so may be considered dated. However, the findings continue to have currency, given the ongoing and increasing attention on the health and wellbeing of NHS staff in contemporary literature, current policies and in the media. For example, the Boorman review of NHS Health and Wellbeing in 2009 recommended that trusts should design and implement strategies for improving the physical and mental health of their staff (DH, 2009). Following this, the NHS Future Forum recommended that Trusts should be held to account for improving the health and wellbeing of their workforce (Ford, 2012). Nurse's health and wellbeing continues to be at the forefront of NHS employee health (Blake, 2014), especially given the high levels of sickness absenteeism, stress and burnout amongst frontline care staff. The environments in which this 
data was collected continue to generate high levels of stress and emotional burden (e.g. Emergency Departments: Maddineshat et al, 2016; Burns Units: Kellogg et al, 2014)

The self-selecting nature of the sampling strategy and the small number of participants means that care needs to be taken when transfering the findings to other settings. All participants were women and this means that the findings may not reflect the experiences of male nurses. Given these limitations there is a need for further research into the experience of stress for nurses working in different settings, the potential support mechanisms for preventing and managing workplace stress effectively and the barriers and facilitators to accessing these.

\section{Conclusion}

Nurses are broadly positive about utilising CATs for their own health and wellbeing, and the provision of wellbeing schemes within the workplace that include CATs is seen as a positive development. Of particular importance is the recognition this provides to nurses that they are valued by their employer. Some logistic and practical issues need to be addressed to ensure that CATs are perceived to be accessible. Workplace culture around taking breaks needs to be further investigated to ensure that nurses feel able to take breaks, and able to access services and that this is supported by their colleagues and managers. Healthcare employers should consider provision of wellbeing services within clinical settings and providing quiet spaces for relaxation and time out during breaks.

\section{References}

Adam, J. (2006) An exploratory study of complementary and alternative medicine in hospital midwifery: Models of care and professional struggle. Complementary Therapies in

Clinical Practice 12, 40-47. 
Adriaenssens, J. \& De Gucht, V. (2015) Causes and consequences of occupational stress in emergency nurses: A longitudinal study. Journal of Nursing Management 23 (3), 346-358.

Begat, J. Ellefsen, B. \& Severinsson, E. (2005) Nurses' satisfaction with their work environment and the outcomes of clinical nursing supervision on nurses' experiences of wellbeing: A Norwegian study. Journal of Nursing Management 13, 221-230.

Blake, H and Batt, ME (2015). Employee perceptions of pedometer walking intervention in a hospital workplace. International Journal of Health Promotion and Education.

DOI:10.1080/14635240, Published Online 02 Mar 2015.

Blake, H, Bennett, E and Batt, ME (2014). Evaluation of occupational health checks for hospital employees International Journal of Workplace Health Management. 7(4), 247266

Blake, H, (2014). Nurses recognise their own health can affect care quality Nursing Times. 110(38), 9

Blake, H, Zhou, D and Batt, ME, 2013. Five year workplace wellness intervention in the NHS Perspectives in Public Health. 133(5):262-71.

Blake, H. \& Lloyd, S. (2008) Influencing organizational change in the NHS: lessons learned from workplace wellness initiatives in practice. Quality in Primary Care. 16(6) 449-455.

Blake, H. Zhou, D. \& Batt, ME. (2013) Five year workplace wellness intervention in the NHS. Perspectives in Public Health. 133(5) 262-271.

Department of Health. (2009). NHS Health and Wellbeing Review [Dr Steve Boorman]. 23 November 2009. Available at: http://webarchive.nationalarchives.gov.uk/20130107105354/http:/www.dh.gov.uk/en/Publica 
tionsandstatistics/Publications/PublicationsPolicyAndGuidance/DH_108799 (Last accessed $31.10 .2016)$

Duffy, E. Avalos, G. \& Dowling, M. (2015) Secondary traumatic stress among emergency nurses: A cross-sectional study. International Emergency Nursing 23 (2): 53-58.

Ford, S. (2012) Nurse health and wellbeing should be 'core principle' for NHS trusts. Nursing Times, 10 January 2012. Available at: https://www.nursingtimes.net/nurse-health-andwellbeing-should-be-core-principle-for-nhs-trusts/5040004.article (Last accessed 31.10.2016)

Furnham, AF. (2001) Alternative and complementary healing practices. IN Smelser, NJ. Wright, J. \& Baltes, PB. (eds) International Encyclopedia of the Social and Behavioural Sciences. Pergamon: Oxford.

Gardner, G. Collins, C. Osborne, S. Henderson, A. \& Eastwood, M. (2009) Creating a therapeutic environment: A non-randomised controlled trial of a quiet time intervention for patients in acute care. International Journal of Nursing Studies 46, 778-786.

Health and Safety Executive (2014) Work Related Stress, Anxiety and Depression Statistics in Great Britain 2015. HSE: London.

Helps, S. (1997) Experiences of stress in accident and emergency nurses. Accident and Emergency Nursing 5(1) 48-53.

Kellogg MB, Barker M, McCune N. (2014) The lived experience of pediatric burn nurses following patient death. Pediatr Nurs. 40(6):297-301.

Lee, S. Blake, H. \& Lloyd, S. (2010) The Price is Right: Making Workplace Wellness Financially Sustainable. International Journal of Workplace Health Management. 3(1) 58-69. 
Lee, S. \& Blake, H. (2009) Tackling NHS staff stress levels in an 'eggstraordinary' way. Health Psychology Update. 18(2) 8-13.

Mackereth, P. White, K. Cawthorn, A. \& Lynch, B. (2005) Improving stressful working lives: Complementary therapies, counselling and clinical supervision for staff. European Journal of Oncology Nursing 9, 147-154.

McVicar, A. (2003) Workplace stress in nursing: A literature review. Journal of Advanced Nursing. 44 (6): 633-642.

Maddineshat M, Rosenstein AH, Akaberi A, Tabatabaeichehr M. (2016) Disruptive Behaviors in an Emergency Department: the Perspective of Physicians and Nurses. J Caring Sci. 1;5(3):241-249. eCollection 2016

Meade, O. Maclennan, SJ. Blake, H. \& Coulson, N. (2009) Workplace complementary and alternative therapies for hospital-site staff. International Journal of Workplace Health Management. 2(3) 258-271.

National Centre of Complementary and Alternative Therapies (2011) Complementary and Alternative Medicine [online]. Available at:

http://nccam.nih.gov/health/providers/camterms.htm [Accessed on: 01/12/2015].

Negble, M. Agbenorku, P. \& Hoyle-Williams, PE. (2014) Nursing severe burn injury patients: Emotional impact on nurses. International Journal of Medicine and Medical Sciences 47 (1): 1430-1433.

NHS Employers (2014) Guidance on the Prevention and Management of Stress at Work. NHS Employers: London. 
NHS England (2015). Simon Stevens announces major drive to improve health in NHS workplace. 02 September 2015. Available at: https://www.england.nhs.uk/2015/09/nhsworkplace/ (accessed on: 07/02/2016).

NHS Improvement (2016) Evidence from NHS Improvement on Clinical Staff Shortages: A Workforce Analysis. NHS Improvement: London.

Peters, D. (2000) From holism to integration, is there a future for complementary therapies within the NHS. Complementary Therapy Nursing Midwifery 6, 59-60.

Phillips, S. (1996) Labouring the emotions: expanding the remit of nursing work? Journal of Advanced Nursing 24, 139-143.

Ritchie, J. \& Spencer, L. (1994) Qualitative data analysis for applied policy research. IN Bryman, A. \& Burgess, RG. (eds.) Analyzing Qualitative Data. Routledge: London. P 173194.

Tiran, D. \& Chummun, H. (2004) Complementary therapies to reduce physiological stress in pregnancy. Complementary Therapies in Nursing and Midwifery 10, 162-167.

Wu, H. Chi, H. Chen, L. Wang, L. \& Jin, Y-P. (2010) Occupational stress among hospital nurses: A cross-sectional survey. Journal of Advanced Nursing 66 (3) 627-634. 